\title{
Depression and anxiety in epilepsy: the association with
} demographic and seizure-related variables Vasilios Kimiskidis*1, Nikolaos Triantafyllou², Eleni Kararizou ${ }^{2}$, StergiosStylianos Gatzonis ${ }^{3}$, Konstantinos Fountoulakis ${ }^{4}$, Anna Siatouni², Panagiotis Loucaidis ${ }^{2}$, Dimitra Pseftogianni ${ }^{1}$, Nikolaos Vlaikidis ${ }^{1}$ and George Kaprinis 4

Address: ${ }^{1}$ Aristotle University of Thessaloniki, Department of Neurology III, Thessaloniki, Greece, ${ }^{2}$ University of Athens, Neurological Clinic, Eginition Hospital, Athens, Greece, ${ }^{3}$ University of Athens, Department of Neurosurgery, Athens, Greece and ${ }^{4}$ Aristotle University of Thessaloniki, Department of Psychiatry III, Thessaloniki, Greece

* Corresponding author

from International Society on Brain and Behaviour: 3rd International Congress on Brain and Behaviour

Thessaloniki, Greece. 28 November - 2 December 2007

Published: 17 April 2008

Annals of General Psychiatry 2008, 7(Suppl I):S32I doi:I0.1186/I744-859X-7-SI-S32I

This abstract is available from: http://www.annals-general-psychiatry.com/content/7/SI/S32 I

(C) 2008 Kimiskidis et al.; licensee BioMed Central Ltd.

\section{Background}

Depression and anxiety are common psychiatric symptoms in patients with epilepsy exerting a profound negative effect on health-related quality of life. Several issues, however, pertaining to their association with psychosocial, seizure-related and medication factors, remain controversial. Accordingly, the present study was designed to investigate the association of interictal mood disorders with various demographic and seizure-related variables in patients with newly-diagnosed and chronic epilepsy.

\section{Materials and methods}

We investigated 201 patients with epilepsy (51.2\% males, mean age $33.2 \pm 10.0$ years, range $=16-60$ ) with a mean disease duration of $13.9 \pm 9.5$ years. Depression and anxiety were assessed in the interictal state with the Beck Depression Inventory, 21 item version (BDI-21) and the State and Trait subscales of the State-Trait Anxiety Inventory (STAI-S and STAI-T), respectively. The association of mood disorders with various variables was investigated with simple and multiple linear regression analyses.

\section{Results}

High seizure frequency and symptomatic focal epilepsy (SFE) were independent determinants of depression, together accounting for $12.4 \%$ of the variation of the BDI21 . The STAI-S index was significantly associated with the type of epilepsy syndrome (SFE). Finally, high seizure frequency, SFE and female gender were independent determinants of trait-anxiety accounting for $14.7 \%$ of the variation of the STAI-T.

\section{Conclusions}

Our results confirm the prevailing view that depression and anxiety are common psychological disorders in epileptics. It is additionally concluded that female gender, high seizure frequency and a symptomatic epilepsy syndrome are independent risk factors for the development of anxiety and/or depression. 